\title{
Formal Operations: A Systematic Reformulation
}

\author{
JAMES P. BYRNES \\ University of Michigan
}

\begin{abstract}
In the present paper it is argued that the original model of formal operations failed to make a clear distinction between the organization of knowledge and the ability to solve tasks. Additionally, too much emphasis was placed on the scientific reasoning aspect of formal operations in previous accounts. The present paper proceeds by first describing a recasted model of formal operations which retains only its essential, nondeletable aspects. Relevant empirical literature is reviewed within the perspective of this recasted model. Then, some positive heuristics, i.e., methodological approaches designed to avoid anomalous results, are proposed as direct tests of this model. Finally, the similarity and differences between the present account and other accounts of formal operations are discussed. 1988 Academic Press, Inc.
\end{abstract}

Current thinking regarding the stage of formal operations is that the model has lost a good deal of its explanatory value (e.g., Braine \& Rumain, 1983; Johnson-Laird, 1983; Keating, 1980; Strauss \& Kroy, 1977). For the most part, the decline in interest in the formal operations model can be linked to the shift in paradigms or research programs (Kuhn, 1962; Lakatos, 1971; Overton, 1984) away from Piagetian theory in general, which emphasizes the generality of structures, toward views of conceptual development which explain age differences in terms of domain specificity and the acquisition of expertise (e.g., Carey, 1985; Chi, Glaser \& Rees, 1982; Keil, 1985). This shift is particularly important for the formal operations model because one of the specific advances of formal thought over concrete thought has been proposed to be the capacity to reason about any content whatsoever (Inhelder \& Piaget, 1969). Additionally, the model has lost favor because of some evidence which shows that not all adolescents and adults demonstrate formal operational reasoning either on Inhelder \& Piaget's (1958) tasks (Keating, 1980; Neimark, 1979) or on related measures like Wason's (1966) Four-Card task (Wason, 1983). A further problem is that the logic itself and Inhelder \& Piaget's explanation of it contain several apparent contradictions, specifically regarding the concept of equivalence (Braine \& Rumain, 1983; Ennis, 1975; Leiser, 1982).

The author thanks Karen Bartsch, Liz DeGroot, Karen Ebeling, and Jacqui Wooley for helpful comments on earlier drafts of this paper. He also thanks David Moshman and Daniel Keating for extremely useful criticisms and cogent comments. Reprint requests should be addressed to James P. Byrnes, Developmental Psychology, University of Michigan, Ann Arbor, MI 48109. 
Recent philosophers of science have explained the historical acceptance or rejection of theoretical paradigms in terms of positive heuristics, or the ability of a theory to anticipate and avoid potentially falsifying instances (Lakatos, 1971). More specifically, a family of theories, or a research programme, consists of a hard core of philosophical assumptions which are protected from falsification by a belt of auxiliary hypotheses, and also consists of the positive heuristics which outline research strategies designed to avoid the accumulation of falsifying instances. An example of a hard core assumption of Piagetian theory would be that knowledge is neither innate nor learned but is constructed. The constructivist aspect of the theory is never questioned by adherents to the tradition and is protected from falsification by auxiliary hypotheses such as "The source of knowledge is the child's actions on the world." The premise is that one would have to falsify the causal role of actions in the formation of logical structures before one could begin to consider the viability of the constructivist epistemology. Beilin (1985) lists structurefunction analysis, activity, and stages as other candidates for core assumptions and Rowell (1983) proposes equilibration.

Lakatos (1971) argues that the viability of a theory relies upon the ability to avoid mounting falsifying evidence. If too many falsifying instances occur, the scientific community shifts to models that better exhibit the capacity to explain anomalies. This view seems to descriptively account well for the shift away from the Piagetian model, although Laudan (1977) criticizes Lakatos' position for, among other things, not specifying how many falsifying instances are necessary for the rejection of a given theory.

The present paper is an attempt to recast the Formal Operations model in light of current criticisms in such a way so as to retain the viable aspects of the model, and to also point out which aspects lead to anomalies and are, hence, dispensable. The paper proceeds by first proposing essential aspects of the formal operations model which must be retained in order that it be consistent with the rest of the theory. As each essential aspect is described, current research which addresses this aspect is introduced. Finally, some positive heuristics and suggestions for future research are discussed.

\section{ESSENTIAL ASPECTS OF FORMAL OPERATIONAL THOUGHT}

Inhelder and Piaget (1958) and Piaget (1972) provide the most extensive treatments of the formal operations logic and can be used to consider the fundamental and useful aspects of this level of thought. In the present analysis, Piaget's (1972) Essai de Logique Operatoire is considered to be the most accurate and consistent treatment of the nature of the logic since 
it contains many of the revisions of the model after the 1950s. The present thesis, therefore, relies most heavily on this work. Inhelder and Piaget's (1958) The Growth of Logical Thinking from Childhood to Adolescence is considered here to be a "content" book focused on how the acquired propositional logic can be applied in scientific problem-solving situations. Support for this view comes from the fact that Inhelder gathered the experimental data for the 1958 book independently of Piaget's theoretical formalization of the formal operations model (Inhelder \& Piaget, 1958, p. xxi).

What follows is my own analysis of the essential aspects of formal operations that is based on the above two works, as well as on other translated and untranslated Genevan studies which address formal reasoning. Similar to Beilin (1985), I consider a given aspect to be essential if it meets two criteria. The first is whether the aspect has had a long history in Piagetian theory and has survived Piaget's own revisions of his theory from the 1920s up to the late 1970s. The second is Beilin's (1985) deletion criteria. Here, an aspect is to be retained if deleting it from the theory would produce substantial changes that would make the theory no longer recognizable as Piaget's. This deletion criteria is, of course, largely intuitive, but can be supported with an appeal to the internal consistency of the theory; that is, if the aspect has analogs at the sensorimotor, preoperational, or concrete operational levels, it should be retained.

It should be pointed out that as far as I know, Piaget never disavowed in writing any of the claims he made in Inhelder and Piaget (1958), though there is reason to believe that he did become disenchanted with using the formal operations model to explain scientific reasoning in later works. First, he never reintroduced the model to explain causal reasoning in Piaget (1974) which represented a compilation of over 100 studies of causal reasoning. Second, it is reasonable to assume that he was quite affected by the noted philosopher E. Beth's attack of the model since he cowrote a concilatory book with Beth years later (i.e., Beth \& Piaget, 1966). Moreover, since Piaget rarely made references to older, weaker versions of his theory in the process of introducing apparent changes in it, one often has to make inferences about problems in old models on the basis of new changes. Hence, the following recasted model is largely my interpretation of the viable aspects of the theory.

A further, and important preliminary point is that the present formulation and review of relevant literature is radically different from other current reviews of formal operational reasoning like Neimark (1979) or Keating (1980). In the present analysis, I make an a priori distinction between the organization of knowledge at a given level of development and the knowledge of how to solve a task. This is similar to Ryle's distinction between "knowing that" and "knowing how" (Ryle, 1971). It is 
argued here that at every level, Piaget's models of structures (e.g., formal operations) represent the organization of knowledge in the mind of the child (i.e., "knowing that"). As a result of this organization, it is argued that children employ specific task solution strategies (e.g., formal operational schemata) to solve the tasks presented to them (i.e., "knowing how"). An example which can illustrate the distinction and relation between "knowing that" and "knowing how" is the relation between children's comprehension of order and their ability to seriate at the level of concrete operations. Concrete operational children understand that an object can be both bigger than one object and smaller than another at the same time, due to the "grouping" of "primary addition of relations" (Flavell, 1963). Piaget formalized this structural understanding ("knowing that") in his notion of "grouping." This concept is the source of the task solution strategy ("knowing how") of holding the sticks in one hand and selecting the largest (or smallest) stick remaining in order to complete the array.

When one attempts to find such a straightforward relation at the formal level, however, it becomes clear that Inhelder and Piaget (1958) failed to keep the two forms of knowledge distinct. In particular, the structural model they chose (i.e., the 16 binary operations of propositional logic) should be used to formalize the organization of knowledge about object properties, classes, etc. Hence, the 16 binary operations are a formalization of "knowing that." Throughout the 1958 book, however, the propositions are often used to formalize the schemata or task solution strategies ("knowing how"). I argue that this is a misapplication of the propositional logic which has lead to conceptual difficulties in the theory as well as an overemphasis in the American literature on the formal operational schemata. In addition, Hintikka (1975) shows how "knowing that" knowledge is not reducible to "knowing how" knowledge, thereby demonstrating that these two forms of knowledge are logically distinct. Therefore the "knowing how" aspect of formal operations is best left to structural models such as Siegler's (1976) rules for the balance task. An additional problem is that, unlike the above seriation example, there is no clear, specified relation between the knowledge which the binary operations represent and the schemata such as proportionality to be found in Inhelder and Piaget (1958).

It is preliminarily proposed here that "knowing that" knowledge allows for setting up goals of task solution which are based on an intuitive notion of what form the answer will probably take, and that the "knowing how" knowledge for a given task is acquired through trial and error or instruction. This characterization cannot account, however, for cases where subjects know how to solve problems with proper rote strategies but fail to comprehend when to apply the solution strategies or how the problem 
fits into a conceptual framework (e.g., Chi et al.'s (1982) physics novices or undergraduates in a statistics course). Therefore, the precise relation between "knowing how" and "knowing that" knowledge at the level of formal operations remains to be specified. While this is an important topic, it is outside the scope of the present paper.

The present review considers the essential aspects of formal operations with respect to the organization of knowledge. Neimark (1979) or Keating (1980), on the other hand, largely review studies which have essentially attempted to verify the existence of schemata such as isolation of variables and proportionality. These studies are not reviewed in the present paper because it is argued that they only indircetly assess the essential aspects proposed here. I come back to this point later.

With all this in mind, I turn now to a discussion of the essential aspects of the formal operations model. These aspects can be listed as a series of postulates: (a) formal operations are operations on operations; (b) formal operations permit the construction of all possible combinations of object attributes; (c) the formal operations model is a propositional representation consisting of the relations among the 16 binary operations as form and statements about class relations or properties serving a "bilevel" content; and (d) formal operations permit hypothetical thinking due to the ability to reason about statements referring to properties of objects rather than simply reasoning directly about objects. It is from these essential aspects that the correlative schemata such as isolation of variables and proportions are derived. I leave aside the role of these schemas in order to describe each of the essential aspects in turn.

\section{OPERATIONS ON OPERATIONS}

The aspect of operations on operations derives from Piaget's (1972) definition of "structure" as "every link capable of playing, alternatively or simultaneously, the role of form and the role of content" (p. 40). This means that each cognitive level in the theory can be characterized by structures that have unique form and unique content. Additionally, the form of a given level becomes the content of the next level. Hence, the form of the sensorimotor level (i.e., schemes) become the content of preoperational thought (i.e., mental imagery). The same is true for the concrete and formal operational levels. If the form of concrete operations consists of inclusion relations, then the form of formal operations consists of inclusions of inclusions (Piaget et al., 1977).

Significant insights are gained within knowledge domains when second order operations like inclusion of inclusions are constructed at the formal operations level. This is particularly so regarding the role of negation (Piaget et al., 1977). More specifically, negation consists of the simple 
complementarity of classes at the concrete operational level when classes are organized around inclusion relations; that is, a subclass such as $A$ and it's complement $A^{\prime}$ are related through the superordinate category $B$. If $B=$ "dogs," $A=$ "terriers," and $A^{\prime}=$ "non-terrier dogs," the elements of class $A^{\prime}$ are those objects which possess properties characteristic of "dogs" but do not possess the properties which are unique to "terriers." Thus, negation so construed means simply complementarity within a given inclusion relation.

Eventually, however, the inclusion relations at a given level become integrated together through the subordination of these classes to an even higher superordinate category (hence, the inclusion of inclusions). This new development is depicted in Table 1. This simple example shows how the classes of "cats" and "dogs" can be united through the superordinate category of "mammals." In effect, the two inclusion structures of "dogs" and "cats" (i.e., $\mathrm{A}+\mathrm{A}^{\prime}=\mathrm{B}$ and $\mathrm{C}+\mathrm{C}^{\prime}=\mathrm{D}$ ) become further nested into a higher superordinate category. What this means is that whereas negating class $A$ prior to this structuration resulted in simply the class $A^{\prime}$, now negation consists of the class $A^{\prime}$ plus those elements under $D$ (Piaget et al., 1977).

As will be discussed in a later section, a formal operational subject can access this organization of knowledge by way of propositions or statements. A new class of inferences is made possible when the child constructs a propositional representation of these inclusions of inclusions. Take, for example, the inferences around a statement of implication such as "if it's a terrier then it's a dog." Negation of the antecedent of the statement $(p)$, "it's a terrier", results in "it's not a terrier" (not-p). Arranging the arguments into a syllogism, one obtains as a major premise "If it's a terricr then it's a dog" and as a minor premise "It's not a terrier." Given these premises, one cannot tell whether the conclusion is "it's a

TABLE 1

Two Inclusions Nested within a Higher Superordinate Category

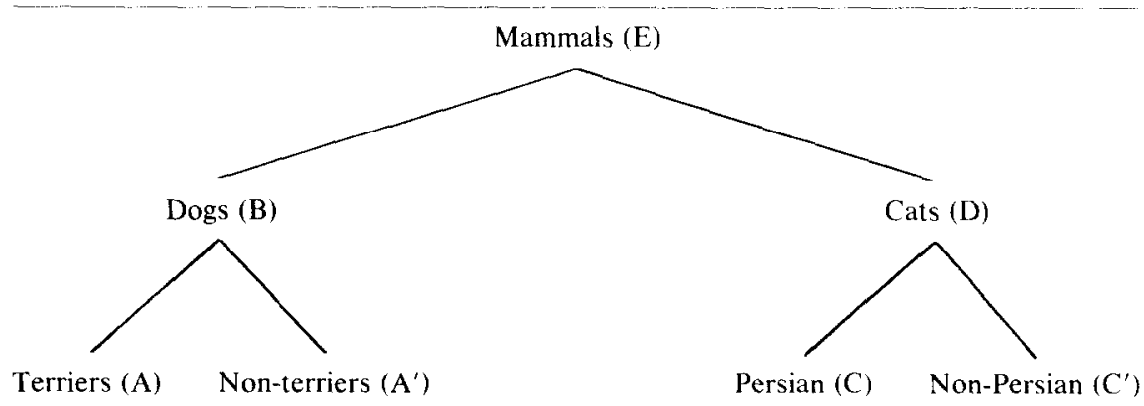


non-terrier dog" or "it's a non-dog." Hence, there is inherent indeterminacy. The correct response, then, is "can't tell." Prior to this structurization, the Piagetian model would predict that children would not say "can't tell" but would say either that it's a non-terrier dog (e.g., "it's a collie") or it's a non-dog (e.g., "it's a cat") because these two alternatives have not been integrated together in a nested inclusion. Such a prediction is obviously testable. Thus, the correct response is provided by the construction of operations on operations.

There have been relatively few studies which can be considered to have directly addressed the aspect of nested inclusions. One study which has considered the changes in the meaning of negation as a result of nested inclusions is Voelin-Liambey and Berthoud-Papadropoulou (in Piaget et al., 1977). The results from this study showed that (a) the appreciation of indeterminate inferences about statements of implication was initially apparent in 10-to 11-year-olds and (b) simple class-inclusion reasoning was insufficient for correct performance. As described above, the correct "can't tell" response is provided by interrelating two inclusions such as (a) mammals $=$ dogs + non-dogs and (b) non-dogs $=$ non-dog mammals + non-dog, non-mammals. Byrnes and Overton (1986) found the identical age trends using sufficient causality semantic content within syllogisms. Numerous other studies have shown that the "can't tell" response on syllogisms is generally not found prior to adolescence (see Braine \& Rumain, 1983, for a review). Performance levels are very high when task statements describe familiar semantic content which can be organized through inclusion relations (e.g., sufficient causality), and when feedback procedures which reduce inappropriate construals of "if" are used. These manipulations are only effective, however, beginning around age 10 to 11 . Hence, there is empirical support for the predicted changes in performance on syllogisms which can be explained by the progressive nesting of inclusions. Of course, tasks other than the conditional syllogism would be appropriate for assessing similar changes in knowledge structures and could be based on the notion of operations on operations. As yet few studies have approached developmental change in knowledge structures using this notion.

\section{CONSTRUCTION OF ALL POSSIBLE COMBINATIONS}

The aspect of combinatorial reasoning partially derives from the former aspect of operations on operations; that is, with continued nesting of inclusions, a complete lattice structure can be formed with the universe of possibilities as an upper bound and the empty class as the lower bound (Flavell, 1963). This combinatorial aspect has been more recently couched within a new explanatory mechanism in Piagetian theory called 
constructive generalization (Piaget et al., 1978). Constructive generalization does not consist of the assimilation of new contents to already constituted forms, but rather, refers to the engendering of new forms and new contents, i.e., new structural organizations. An example of constructive generalization is the reciprocal assimilation of two schemes of the same rank. Within this perspective, the universe of possibilities is constructed through the generalization of vicariance relations. Vicariance is a concrete operational ability where the child can take a set of objects and classify them along different dimensions. For example, with a set consisting of both big and small red squares and big and small green circles, the child can group and regroup objects based on color, size, or shape. The formal operational child can consider two or more of these vicariance relations simultaneously, can carry out vicariance operations exhaustively, and can accurately quantify the extension of classes of objects that fall into two or more categories at the same time (Piaget et al., 1978). This ability serves as the basis for the distinction between "and" and "or" and, hence, the understanding of the intersection of classes.

A number of recent studies have considered children's combinatorial reasoning ability (e.g., Douglas \& Wong, 1977; Flexer \& Roberge, 1980; Kuhn \& Angelev, 1976; Lawson, 1977; Martorano, 1977; Neimark, 1975; Roberge, 1976; Roberge \& Flexer, 1979; Saarni, 1973). Almost all these studies show that by the eighth grade (i.e., 13 years old) $80-90 \%$ of subjects accurately generate the various combinations required. However, several studies show that this performance is better for combinations of colored tokens than for the original Inhelder and Piaget (1958) chemicals tasks.

It should be pointed out that whereas the focus of this review is on the organization of knowledge and not on task solution strategies, combinatorial reasoning is considered by Inhelder and Piaget to be one of the formal operational schemata. However, it can be argued that the ability to generate all the pussible combinations very closely reflects the subject's ability to mentally organize the information hierarchically along the combined properties. Additionally, Piaget et al. (1978) present evidence of a progressive development from understanding vicariance at age 7 to 8 , to an understanding of all possible combinations of object properties at age 11 to 12 . Since the results of this study are presented in the form of protocols, it is in need of replication. Nevertheless, there is substantial support for this structural prediction.

\section{PROPOSITIONAL REPRESENTATION}

In addition to the insights gained due to the acquisition of operations on operations and to the construction of all combinations, there is the emer- 
gence of propositional reasoning at the formal operations level. In particular, the class knowledge acquired at the level of concrete operations becomes reorganized and accessible by way of a network of propositions about this acquired knowledge. In order to make precise what propositional reasoning entails, it is best to begin with a few definitions. For Piaget (1972), propositions are

categorical statements, true or false and affirmative and negative . . To say that the statement is categorical excludes imperatives and optatives, but does not exclude the truth or falsity of a proposition that can be proved by virtue of a simple hypothesis. To say that a statement is true or false implies that it has meaning, as opposed to a statement devoid of meaning. (p. 34).

Hence propositions are meaningful statements of which one knows the truth or falsity and through which one reasons about categorical content (e.g., classes or properties). Of course, propositions can also be statements whose truth is merely assumed for the sake of argument such as counterfactual conditionals (Matalon, 1962).

Such propositions can be composed and compared by way of an interpropositional operation which is

every composition which allows one to construct--by means of some propositions ... other propositions well-determined and characterized respectively by the various possible combinations of only those respective truth values. (p. 34)

Table 2 summarizes various interpropositional operations. This table shows that a subject can, for example, compose "it's a terrier" with "it's a dog" into the conditional "If it's a terrier then it's a dog" or the disjunctive "Either it's a dog or it's a terrier." Moreover, transitive relations between propositions (e.g., "if $p$ then $q$; if $q$ then $r$; therefore if $p$ then $r$ ") can be constructed.

TABLE 2

IN I ERPKOPOSITIONAL OPERATIONS

A. Composition e.g., (It's an A) $+\left(\right.$ It's a B) $\rightarrow^{a}$ Either it's an A or B

B. Transitive inferences e.g., (If $p$ then $q$ ) + (If $q$ then $r) \rightarrow($ If $p$ then $r$ )

C. Falsification e.g., (If $p$ then $q)+(p$ and not-q) $\rightarrow$ (If $p$ then $q$ ) is false

D. Equivalence relations e.g., (Either $\mathrm{p}$ or $\mathrm{q})^{b}=($ If not-p then $\mathrm{q})$ $(Q$ if and only if $p)=(\text { Either } p \text { or not-q })^{c}$

\footnotetext{
"The symbol " $\rightarrow$ " is roughly meant to represent "yields" and does not mean strict implication. Also the symbol "+" means "composed with."

${ }^{b}$ Inclusive disjunction.

${ }^{c}$ Exclusive disjunction.
} 
A further important interpropositional operation is negation. Every binary operation is linked uniquely to another binary operation which is its negation. The negation operation represents the cancellation or nullification of a direct operation in that if the direct operation is true, its negation must be false and if the negation is true, then the direct must be false. As shown in Table 2, the truth of the proposition "p and not-q" (e.g., "it is a dog and a non-mammal") would mean that the proposition "if $p$ then q" (e.g., "if it's a dog then it's a mammal") is false. Depicted in terms of classes as in Piaget (1972), the negation operation represents those classes which are excluded from the arrangement of classes specified by the direct operation. It is in this way that the negation operation is the complement of the direct operation under complete affirmation (i.e., the universe of possibilities). Table 3 shows implication and its negation as represented by classes. It is important to note that to construct a direct operation and thus understand which cases are permissible, one must simultaneously construct the negation operation and thus understand which cases are impossible. Hence, the construction of all possible combinations and the construction of the negation operation mutually imply one another.

TABLE 3

IMPLICATION AND ITS NEGATION OPERATION

Direct

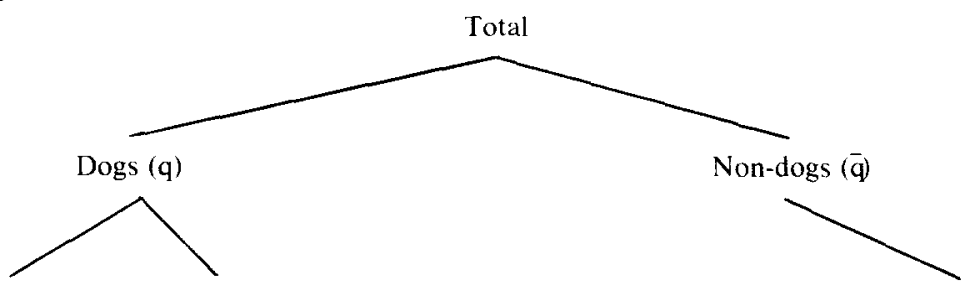

Terriers $(\mathrm{p}) \quad$ Non-terriers $(\overline{\mathrm{p}})$

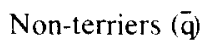

Negation

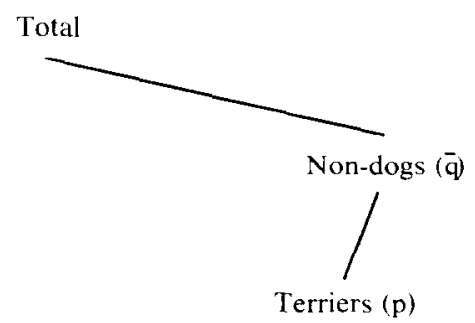


The last important interpropositional operation listed in Table 2 is the formation of equivalence relations among binary operations. Every binary operation can be phrased in multiple equivalent ways. It is in this way that the model specifies that "either p or q" and "if not-p then q" can be used to refer to the same state of affairs between the events described in the propositions " $p$ " and " $q$ " (i.e., a disjunctive relation between " $p$ " and " $q$ ").

It should be pointed out that what makes reasoning at this level formal is that it no longer bears directly on objects, but rather, on statements about objects and the relations between statements, in almost a metalinguistic sensc. The form of formal operations, then, consists of the relations among propositions and the content of these forms are the statements themselves. These statements are, however, categorical and refer to classes or relations among properties of objects. But again, these latter relations are the form of concrete operations. This implies that formal reasoning is neither "pure" nor "abstract" in the sense of making no reference to meaningful content. On the contrary, there is a "bilevel" or nested content consisting of statements at one level, and their content (i.e., that to which they refer) at a lower level.

Regarding the form-content distinction at the formal and concrete levels Piaget (1972) remarks

One of these two domains of logic [i.e., concrete and formal operations] is . . more "pure" or more formalized than the other, but it is question of degree and not of a natural opposition. (p. 37)

Said another way, a concrete operational child would be able to reason about class relations such as the relative extension of subclasses and superordinant classes (e.g., "Are there more red beads or wooden beads?") which bear directly on the objects. The formal operational subject, on the other hand, can reason about interpropositional constructions such as the implication relation between the propositions "it's a terrier" and "it's a dog" contained in the statement "if it's a terrier then it's a dog." Notice that the proposition has meaningful content but is also categorical.

Another test of the attainment of formal operations would be, then, to look for age differences in the ability to draw appropriate inferences about categorical statements. For example, one can employ the syllogism format and require inferences like "If it's a terrier then it's a dog. It's not a terrier. Is it a dog?." Correct performance on such task relies on the ability to reason about these statements but also requires the prior construction of nested inclusions as described above.

A number of studies have directly assessed children's understanding of several of the binary operations using verbal or propositional reasoning 
tasks. On these tasks, subjects are presented with statements constructed with logical connectives (e.g., "if-then" and "either-or") and are asked to either make deductive inferences using these statements as premises, or to make truth judgments about them. The binary operations which have been considered in recent studies include (a) conditional implication (e.g., Byrnes \& Overton, 1986; Moshman, 1977; O'Brien \& Overton, 1980, 1982), (b) disjunction (e.g., Beilin \& Lust, 1975; Moshman, 1977; Neimark \& Slotnick, 1971), (c) conjunction (e.g., Beilin \& I ust, 1975; Neimark \& Slotnick, 1971), and (d) biconditional equivalence (e.g., Byrnes, 1985; Roberge, 1976; Roberge \& Flexer, 1979). These concepts can be expressed by "if-then," "either-or," "and," and "if and only if," respectively. Generally, these studies show that children seem to understand the binary operations associated with these connectives beginning in early adolescence. Performance is optimal when meaningful semantic content (i.e., categorical or causal) and linguistic feedback to elicit the logical meaning of the connectives are used. Such procedures are necessary because there is a poor fit between the natural language interpretations and the logical interpretations of "if" (Geis \& Zwicky, 1971), "either-or" (Neimark \& Slotnick, 1971), and "and" (Braine, 1978). "If"' is commonly interpreted as "if and only if," "either-or" takes on its exclusive rather than inclusive meaning, and "and" is interpreted as indicating a temporal sequence (i.e., " $p$ and then $q$ ") in natural language contexts. Simply pointing out directly or indirectly that the logical meaning is desired greatly improves performance in subjects ages 10-11 and older.

In addition to demonstrating that subjects understand the logical meaning of these connectives, several studies show that subjects comprehend another interpropositional operation, the relation between a binary operation and its negation (Byrnes, 1985; Byrnes \& Overton, 1986; O'Brien \& Overton, 1982; Overton, Byrnes, \& O'Brien, 1985). These studies have shown significant changes between the fourth and the sixth grades in the understanding that only the case " $p$ and not-q" falsifies an "if $p$ then $q$ " hypothesis using Wason's (1966) Four Card task and conditional syllogisms.

A further finding relates to the model's structural description of how all of the binary operations can be expressed in multiple, equivalent ways (see Table 2). For example, "Either it's Tuesday or it's Wednesday" and "If it's not Tuesday, then it's Wednesday" both express the same logical relation and are, hence, logically equivalent to each other (Quine, 1950). Piaget (1972) describes how each of the binary operations stands in such equivalence relations. Basing tasks on this model, Byrnes (1985) found that 10-to 13-year-olds recognized the equivalence of sentence pairs at a rate of $82 \%$ correct.

Perhaps it is best, however, to characterize the propositional reasoning 
of early adolescents as reasoning based on structures which are in the process of becoming consolidated rather than on a consolidated set of structures; that is, there are a multitude of "links" which have to be established between the 16 binary operations (as in Table 2) and it is likely that these links are formed progressively. Moshman (1977), for example, found that the performance of adolescents on a task requiring reasoning about concepts of implication and disjunction follows a pattern of consolidation rather than an abrupt onset. This pattern is entirely consistent with Inhelder and Piaget's (1958) and Piaget's (1972) claims that the formal level consists of an early formatory sublevel and a later consolidated sublevel. Both sublevels are, however, characterized by interpropositional relations as form and statements as content, though in the latter sublevel these relations are completed and integrated. It is the appearance of new forms which reintegrate old forms that makes for the qualitative change between the concrete and the formal levels-not the emergence of a complete and fully integrated system. This characterization implies, then, that the change is by no means abrupt or saltatory.

It must be noted that recent studies have also found that there are some kinds of inferences, such a modus ponens on syllogism tasks, that even 5-year-olds can make quite well (see Braine \& Rumain, 1983, for a review). This finding represents an anomaly for which the present model needs to account. In order to account for this finding, it must be emphasized that none of the essential aspects outlined here should be considered in isolation, as a necessary and sufficient condition for the attribution of formal reasoning. Each of them are, on the contrary, simply necessary criteria which in conjunction represent formal operational thought. Hence, all aspects must be present, but none are sufficient to warrant the attribution of formal reasoning to an individual. Thereforc, the incidence of composing propositions in inferential chains as in modus ponens (i.e., "If $\mathrm{p}$ then q. P therefore q") in kindergartners is insufficient for arguing that these children evidence formal operational reasoning. Such inference making can be considered a precursor ability which becomes incorporated into the subsequent level of formal reasoning.

\section{HYPOTHETICAL REASONING}

A final essential aspect of formal operational thought related to that of propositonal reasoning is the ability to reason hypothetically. More than simply the ability to reason about "if-then" hypotheses, it is argued that the label refers to treating any statement as if it were true, even those which the subject knows in fact are not true (Matalon, 1962). It is because statements are the content of formal thought that hypothetical reasoning becomes possible.

But it is important to point out that in order to reason hypothetically, 
competent formal reasoners in any culture have to be aware of the appropriate linguistic expressions that denote, among other things, counterfactuals and statements about unactualized possibilities. Experimentally, if one wanted to test a subject's ability to reason hypothetically, perhaps the most discriminative and accurate tests would be those which employ counterfactual terms such as "were" and "would be." It is argued that most subjects would not treat a sentence such as "if elephants are coldblooded then they are not mammals" as a counterfactual because of the present indicative tense of the verbs employed. They would be more likely to treat a sentence such as "if elephants were cold-blooded, then they would be not be mammals" as a counterfactual. The subjunctive tense of the verb changes the pragmatic assumptions surrounding the "if" statement (Scholnick \& Wing, 1982; Wing \& Scholnick, 1981). Because subjects have acquired the capacity to reason about propositions and have learned the appropriate marked terms, they are assumed to make a discrimination between these surface expressions. Additionally, they are assumed to cognitively alter the actual physiology of elephants hypothetically so that elephants are attributed a property which is not part of the intension for the class of mammals. Subjects can then assess the entailment relation between the antecedent and the consequent; that is, they would assess whether it is possible for an elephant to be cold-blooded and still be a mammal.

What this description implies, then, is that in testing for hypothetical reasoning it is important to (a) employ content which is modifiable hypothetically, i.e., about which the subject is likely to have organized knowledge, and (b) use the appropriate tense, etc., of the statements within an experiment. There is a tendency, however, in the propositional reasoning domain (c.g., Wason, 1983; Wason \& Johnson-Laird, 1972) to ignore both of these constraints. For example, in studies of conditional reasoning which employ Wason's (1966) Four Card task, the subject is expected to treat the content of sentences like "if there is a D on one side of a card, there is a 7 on the other" as if it were a conditional relation. A conditional relation, however, is one where the antecedent $(p)$ is a sufficient but not necessary condition for the consequent $(q)$ and where the antecedent entails the consequent. This means that if the antecedent is true, then the consequent must be true (entailment) but also that the antecedent is not the only way to obtain the consequent (sufficiency). Two content domains which reflect conditional relations are sufficient causality (e.g., "if it rains then the grass gets wet") and class inclusion relations. The arbitrary content used by Wason and his colleagues cannot be transformed hypothetically into relations such as class inclusion or sufficient causality by analogy because there is no basis for the analogy. Also, it is argued that subjects would only attempt such analogy if the subjunctive tense were employed. It is precisely because the above sentence concerning ele- 
phants taps into existing knowledge and the proper tense is employed that the entailment can be assessed by analogy.

This feature of hypothetical reasoning taken together with the combinatorial aspect forms the additional aspect of the subordination of the real to the possible; that is, that which the subject knows is true about this world becomes a subset of what is logically possible (Inhelder \& Piaget, 1958). Stated another way, the combination of the factual with the counterfactual (as described by the subject's constructed propositions) forms a universe of possibilities. An important difference between the present account and that of Inhelder and Piaget is that possibility is argued here to stem from linguistic-cognitive conversion abilities whereas the latter authors argue that possibility is mostly the result of generating all the possible combinations of object attributes.

Only a handful of studies have empirically addressed the hypothetical reasoning aspect. Meyer (1977) asked 5-, 6-, and 9-year-olds and adults to draw conclusions from counterfactual conditional statements. He found that the 5-and 6-year-olds would not accept the premises due to a strict reality orientation. The 9-year-olds showed less of an adherence to realism, but their reasoning was largely based on a linguistic orientation; that is, they did not reason about hypothetical properties (e.g., oranges that are square) as much as refer to the specific wording of the premise as if it reflected an immutable law. The college students, on the other hand, evidence true contrary-to-fact reasoning in that they accepted the counterfactual premises as logically necessary for argument's sake, and reasoned accordingly. Scholnick \& Wing (1983) found similar results where 12-and 15-year-olds and adults reasoned correctly $65 \%$ of the time on syllogisms using "if" premises in the subjunctive tense. When these subjects were incorrect, they tended to reject conclusions based on the counterfactual, i.e., empirically false, outcome.

In a related fashion, Moshman and Franks (1986) asked subjects to sort "if-then" transitive inference problems which varied on semantic content, empirical truth, and validity dimensions. The valid-only problems were, in effect, counterfactuals (e.g., "If elephants are either animals or plants, and elephants are not animals, then elephants are plants."). Even though the subjunctive tense was not employed for the valid-only problems, $45 \%$ of seventh graders and $85 \%$ of college students sorted problems on the basis of validity whereas no fourth grader did. Also, when asked to rank the three most logical problems, $0 \%$ of the fourth graders, $35 \%$ of the seventh graders, and $61 \%$ of the adults chose the problems involving validity.

\section{SUMMARY OF THE ESSENTIAL ASPECTS}

In summary, then, formal operational reasoning is made possible by the construction of nested inclusions of information (operations on opera- 
tions), the generalization of vicariance into the set of all combinations, and the construction of a propositional representation of acquired knowledge which makes hypothetical reasoning possible. My review of the relevant literature with respect to each aspect shows significant support for the structural predictions made by the model. It should be pointed out, however, that many of the reviewed studies were simply concerned with age trends for specific abilities and were not concerned with verifying the model I have proposed here. Therefore, there is a need for additional studies which directly test each of the proposed structural differences between concrete and formal operational subjects. The positive heuristics to follow are offered as guidelines for testing the structural model of formal operations described here.

\section{POSITIVE HEURISTICS}

As described earlier, positive heuristics outline research strategies meant to avoid anomalies. However, Lakatos (1971) also points out that positive heuristics should advance a given theory so as to better account for a certain class of phenomena. The present section outlines research strategies which are meant to advance a structural study of adolescent reasoning, but are also meant to provide strong tests of the formal operations model.

First, a true test of the model should assess the emergence of specific structural links which make possible a class of inferences which were not evident before. The formal operations model provides examples of these links such as the relation between a direct operation and its negation, the logical equivalence of multiple surface expressions for the same binary operation, and the syllogistic inferences. Tests of these relations are more than assessments of children's comprehension of the semantics of a single expression constructed with logical connectives such as "if" or "eitheror"; They assess the relations between statements or propositions and hence, assess interpropositional reasoning. Methodologically, one should present statements which may or may not refer to observable objects or relations, and require inferences about these statements as well as justifications of these inferences. Such an approach directly addresses propositional reasoning and avoids many of the problems associated with protocol data obtained from Inhelder and Piaget's (1958) tasks. In a related argument, Falmagne (1980) draws a distinction between propositional reasoning tasks and Inhelder and Piaget's "scientific" reasoning tasks. She argues that the latter only become propositional reasoning tasks if the subject encodes the data by way of the appropriate propositions or statements.

Second, one should exploit performance variables and feedback in the 
direction of favoring optimal performance in order to show age trends in the organization and representation of already acquired knowledge. What is unique to the Piagetian approach can be summarized as such: it is not that older children know "more" about a given topic (e.g., classes) but that this knowledge is organized in a different way (e.g., nested inclusions) and this organized knowledge is accessible by way of a new level of representation (e.g., propositions). Age trends, however, can only be shown (or not shown, for that matter) when the content of tasks if familiar to the age groups and when feedback is given which indicates that the logical interpretation of connectives is desired.

Moreover, each of the binary operations specifies a particular organization of knowledge as Table 3 implies. If the primary focus of a given study is to assess age differences in particular levels of organization and representation, then it is necessary to employ semantic content which conforms to these organizations experimentally. For example, one should employ class inclusion or sufficient causality content for conditional reasoning tasks, or content which describes perfectly correlated attributes for biconditional reasoning tasks, etc. Also, the content for a given task should be isomorphic to the taxonomic relations specified in a given culture and should reflect the naturally occurring space-time relations of the content. Violations in these constraints either hinder the detection of or distort age-related trends in organization and representation.

If, on the other hand, the focus is on whether subjects can detect the formal similarities between logical arguments as in Moshman and Franks (1986), then the content can be varied based on the validity, truth, and falsity dimensions.

Finally, as specified above, hypothetical reasoning can only be accurately assessed by employing content which can be altered hypothetically and by employing the proper tense. For example, subjects need to know the intension-extension relations of a given class before they can hypothetically delete properties or treat an object as if it were a member of another class.

All these constraints on content by no means imply that formal operational subjects are reality bound. It is precisely because their organization of "real" information can be accessed and altered by language that thought becomes liberated from objects. To say that thought is liberated from reasoning only about real objects, as in counterfactual reasoning, by no means implies that form and content become "dissociated" at the formal level; it simply means that language provides the means to reason about objects that are hypothetically constructed by analogy.

It should be pointed out that all these heuristics are in accord with Piaget (1962) who argues that the formal operations model is meant to be a model of natural logic and not a model of a formally pure logic. The goal 
was to provide a structural system which accurately describes how adolescents and adults actually reason with meaningful content.

\section{CONCLUSIONS}

At the beginning of this paper, I drew a distinction between "knowing that" and "knowing how" at the level of formal operations. It should be clear at this point that my emphasis on the organization of knowledge is very different than other descriptions of formal operations (e.g., Braine \& Rumain, 1983; Keating, 1980; Strauss \& Kroy, 1977). This is precisely because these authors have all emphasized the scientific problem-solving strategies in their descriptions. This overemphasis is entirely due, I think, to the fact that the only translated work on formal operations available is that of Inhelder and Piaget (1958). It should be noted, however, that the majority of Genevan studies on formal reasoning since the 1958 book such as Beth et al. (1962) and Piaget et al. $(1977,1978)$ have focused on propositional reasoning in "natural contexts" and on changes in the organization of knowledge.

Whereas it is argued in this review that the tasks described in Inhelder and Piaget (1958) only indirectly assess the structural changes described here (since they mostly assess the "knowing how" aspects), Inhelder and Piaget argue that all of their tasks require interpropositional and hypothetical reasoning ability. I am not necessarily disputing this point, but I am arguing that this claim has led to the current rejection of the model. A historical account of the acceptance or rejection of the formal operations model in American psychological circles shows that the key issue is whether one accepts the claim that successful performance on Inhelder and Piaget's tasks necessarily requires subjects to reason via the 16 binary operations. Inhelder and Piaget apparently felt that their claim was strengthened by evidence gained from protocols which they formalized using the 16 binary operations. However, this methodological approach is unsatisfactory for the advancement of the theory for two reasons. The first is that none of the recent studies reviewed in Keating (1980), Meehan (1984), or Neimark (1979) provide the reader with actual protocols. The authors relate that stage scoring is "based on" Inhelder and Piaget (1958). It is very possible that differences in scoring have led to the disparity in reported success rates on these tasks. The second, and more serious reason is that critics of the theory remain entirely unconvinced by the protocols selected by Inhelder and Piaget as supportive of their claims (see, for example, Braine \& Rumain, 1983; Bynum, Thomas, \& Weitz, 1972; Ennis, 1975; Keating, 1980). The argument that opponents seem to make is that since it is possible to come up with other apparently adequate models of reasoning, and since the protocol data is dubious, one need not accept the claim that the 16 binary operations so defined are necessary for 
successful solution of these tasks. From a philosophy of science standpoint, it would seem that Inhelder and Piaget's (1958) choice of tasks has led to an apparent inability to explain anomalies and to a desire to reject the model. From a Piagetian standpoint, the need for more fruitful positive heuristics is clear. It is my contention that the model proposed here has generated and will continue to generate successful research strategies.

Some final comments are in order regarding what aspects of formal operational thought that I have not considered to be essential. First, I made no specification regarding the role of age in the model. My interpretation of numerous Piagetian works is that the finding that children typically evidence many aspects of formal operations at around age 11 is a contingent and nonnecessary empirical fact. It is interesting but nonessential that children move through the stages in many content domains and enter the formal stage at around 11, but theoretically and empirically it is possible to find formal operational subjects at a lower age. Therefore, a child's age is a nonessential aspect of formal operations. Second, there is no need to retain the notion of "cross-domain generality" in order to adopt the present (or former) model of formal operations. One need only require that the order of cognitive development proceed from sensorimotor to formal operations for every content domain with which the child has experience. Hence, it is possible to be "formal" in some areas and "concrete" in others. It is my contention that once a child uses the 16 binary operations to construct a propositional representation of a given content (which can also be characterized by the other essential aspects), this child can be said to have entered the level (not "stage") of formal operations for that domain. The key criterion, then, is the nature of the form and content as specified earlier in this paper. It is unlikely, however, that children construct knowledge (or "gain expertise") in only one domain, and that it is likely that many related domains are constructed at the formal level simultaneously. Regardless, the notion of cross-domain generality is also a deletable construct. An extended justification for the above deletions is obviously in order, but is outside the scope of this paper.

In summary, I am arguing that the adoption of the formal operations model proposed here and the deletion of the notions of "contentless" reasoning, age as an essential component, and cross-domain generality make for a significantly more viable and accurate model of adolescent reasoning than the former model of formal operations. The next obvious step is to integrate the present recasted model of "knowing that" with current models of "knowing how."

\section{REFERENCES}

Beilin, H. (1985). Dispensable and indispensable elements of Piaget's theory: On the core of 
Piaget's research program. In J. Montangero (Ed.), Genetic epistemology: Yesterday and today. New York: CUNY press.

Beilin, H., \& Lust, B. (1975). A study of the development of logical and linguistic connectives. In H. Beilin (Ed.), Studies of the cognitive basis of language development (pp. 217-337). New York: Academic Press.

Beth, E. W., Grize, J.-B., Martin, R., Matalon, B., Naess, A., \& Piaget, J. (1962). Implication, formalisation et logique naturelle (Vol. 16, Etudes D'Epistemologie Genetique). Paris: PUF.

Beth, E. V., \& Piaget, J. (1966). Mathematical epistemology and psychology. Dordrecht: Reidel.

Braine, M. D. S. (1978). On the relation between the natural logic of reasoning and standard logic. Psychological Review, 85, 1-21.

Braine, M. D. S., \& Rumain, B. (1983). Logical reasoning. In P. Mussen (Ed.) Handbook of child psychology (Vol. 3, pp. 263-340). New York: Wiley.

Bynum, T. W., Thomas, J. A., \& Weitz, L. J. (1972). Truth-functional logic in formal operational thinking: Inhelder and Piaget's evidence. Developmental Psychology, 7 , 129-132.

Byrnes, J. P. (1985). The development of the conditional and biconditional. Unpublished Doctoral dissertation, Temple University.

Byrnes, J. P., \& Overton, W. F. (1986). Reasoning about certainty and uncertainty in concrete, causal and propositional contexts. Developmental Psychology, 22, 793-799.

Carey, S. (1985). Conceptual change in childhood. Cambridge, MA: MIT press.

Chi, M. T. H., Glaser, R., \& Rees, E. (1982). Expertise in problem solving. In R. Sternberg (Ed.) Advances in the psychology of intelligence (Vol. 1, pp. 7-75). Hillsdale, NJ: Erlbaum.

Douglas, J. D., \& Wong, A. C. (1977). Formal operations: Age and sex differences in Chinese and American children. Child Development, 48, 689-692.

Ennis, R. H. (1975). Children's ability to handle Piaget's propositional logic: A conceptual critique. Review of Educational Research, 45, 1-41.

Falmagne, R. J, (1980). The development of logical competence: A psycholinguistic perspective. In H. Spada \& R. H. Kluwe (Eds.), Developmental models of thinking (pp. 171-197). New York: Academic Press.

Flavell, J. H. (1963). The developmental psychology of Jean Piaget. New York: Van Nostrand.

Flexer, B. K., \& Roberge, J. J. (1980). IQ, field dependence-independence, and the development of formal operational thought. Journal of General Psychology, 103, 191-201.

Geis. M., \& Zwicky, A. M. (1971). On invited inferences. Linguistic Inquiry, 2, 561-566.

Hintikka, K. J. J. (1975). Different constructions in terms of the basic epistemological verbs. In K. J.J. (Ed.), The intentions of intentionality and other new models for modalities. Dordrecht: Reidel.

Inhelder, B., \& Piaget, J. (1958). The growth of logical thinking from childhood to adolescence. New York: Basic Books.

Inhelder, B., \& Piaget, J. (1969). The psychology of the child. New York: Basic Books.

Johnson-Laird, P. N. (1983). Thinking as a skill. In J. St. B. T. Evans (Ed.), Thinking and reasoning: Psychological approaches (pp. 164-196). London: Routledge \& Kegan Paul.

Keating, D. P. (1980). Thinking processes in adolescence. In J. Adelson (Ed.), Handbook of adolescent psychology (pp. 211-246). New York: Wiley.

Keil, F. C. (1985). On the stage-dependent nature of stages of cognitive development. In I. Leven (Ed.), Stage and structure revisted. Princeton: Ablex.

Kuhn, D., \& Angelev, J. (1976). An experimental study of the development of formal operational thought. Child Development, 47, 697-766. 
Kuhn, T. (1962). The structure of scientific revolutions. Chicago: Univ. of Chicago Press. Lakatos, 1. (1971). Falsification and the methodology of scientific research programmes. In I. Lakatos \& A. Musgrave (Eds.), Criticism and the growth of knowledge (pp. 91-180). Cambridge: Cambridge Univ. Press.

Laudan, L. (1977). Progress and its problems: Toward a theory of scientific growth. Berkeley, CA: Univ. of California Press.

Lawson, A. E. (1977). Relationship among performances on three formal operations tasks. Journal of Psychology, 96, 235-241.

Leiser, D. (1982). Piaget's logical formalism for formal operations: A interpretation in context, Developmental Review, 2, 87-99.

Martorano, S. C. (1977). A developmental analysis of performance on Piaget's formal operations tasks. Developmental Psychology, 13, 666-672.

Matalon, B. (1962). Étude genetique de l'implication. In E. W. Beth et al. (Eds.), Implications, formalisation et logique naturelle (Vol. 16, Études d'epistemologie genetique). Paris: PUF.

Meehan, A. M. (1984). A meta-analysis of sex differences in formal operational thought. Child Development, 55, 1110-1124.

Meyer, J. S. (1977). The development of contrary-to-fact reasoning. Paper presented at the Biennial Meeting of the Society for Research in Child Development, New Orleans.

Moshman, D. (1977). Consolidation and stage formation in the emergence of formal operations. Developmental Psychology, 13, 95-100.

Moshman, D., \& Franks, B. A. (1986). The development of the concept of inferential validity. Child Development, 57, 153-165.

Neimark, E. D. (1975). Longitudinal development of formal operations thought. Genetic Psychology Monographs, 91, 171-225.

Neimark, E. D. (1979). Current status of formal operations research. Human Development, 22, 60-67.

Neimark, E. D., \& Slotnick, N. S. (1971). Development of the understanding of logical connectives. Journal of Experimental Psychology, 61, 451-460.

O'Brien, D. P., \& Overton, W. F. (1980). Conditional reasoning following contradictory evidence: A developmental analysis. Journal of Experimental Child Psychology, 30, 44-60.

O'Brien, D. P., \& Overton, W. F. (1982). Conditional reasoning and the competenceperformance issue: A developmental analysis of a training task. Journal of Experimental Child Psychology, 34, 274-290.

Overton, W. F. (1984). World views and their influence on psychological theory and researclı: Kulı-Lakatos-Laudan. In H. W. Reese (Ed.), Advances in child development and behavior (Vol. 18, pp. 191-226). New York: Academic Press.

Overton, W. F., Byrnes, J. P., \& O’Brien, D. P. (1985). Developmental and individual differences in conditional reasoning: The role of contradiction training and cognitive style. Developmental Psychology, 21, 692-701.

Piaget, J. (1962). Introduction. In E. W. Beth et al. (Eds.), Implication, Formalisation et Logique Naturelle (Vol. 16, Études d'epistemologie genetique). Paris: PUF.

Piaget, J. (1972). Essai de logique operatoire: Deuxieme édition du traite de logique. Paris: Dunod.

Piaget, J. (1974). Understanding causality. New York: Basic Books.

Piaget, J., et al. (1977). Recherches sur l'abstraction reflechissante (Vol. 34. Études d'epistemologie genetique). Paris: PUF.

Piaget, J., et al. (1978). Recherches sur al generalisation (Vol. 37 Études d'epistemologie genetique). Paris: PUF.

Quine, W. V. O. (1950). Methods of logic. New York: Holt, Rinehart \& Winston. 
Roberge, J. J. (1976). Developmental analyses of two formal operational structures: Combinatorial reasoning and conditional reasoning. Developmental Psychology, 12, 563564.

Roberge, J. J., \& Flexer, B. K. (1979). Further examination of formal operational reasoning abilities. Child Development, 50, 478-484.

Rowell, J. A. (1983). Equilibration: Developing the hard core of the Piagetian research program. Human Development, 26, 61-71.

Ryle, G. (1971). Knowing how and knowing that. In G. Ryle (Ed.), Collected papers (Vol. 2, pp. 212-225). New York: Barnes \& Noble.

Saarni, C. I. (1973). Piagetian operations and field independence as factors in children's problem-solving performance. Child Development, 44, 338-345.

Scholnick, E. K., \& Wing, C. S. (1982). The pragmatics of subordinating conjunctions: A second look. Journal of Child Language, 9, 461-479.

Scholnick, E. K., \& Wing, C. S. (1983). Evaluating presuppostions and propositions. Journal of Child Language, 10, 639-660.

Siegler, R. S. (1976). Three aspects of cognitive development. Cognitive Psychology, 8, $481-520$.

Strauss, S., \& Kroy, M. (1977). The child as logician or methodologist? A critique of formal operations. Human Development, 20, 102-117.

Wason, P. C. (1966). Reasoning. In B. M. Foss (Ed.), New horizons in psychology. Harmondsworth, England: Penguin.

Wason, P. C. (1966). Realism and rationality in the selection task. In J.St.B.T. Evans (Ed.), Thinking and reasoning: Psychological approaches (pp. 44-75). London: Routledge \& Kegan Paul.

Wason, P. C.. \& Johnson-Laird, P. N. (1972). Psychology of reasoning: Structure and content. Cambridge: Harvard Univ. Press.

Wing, C. S., \& Scholnick, E. K. (1981). Children's comprehension of pragmatic concepts in 'because', 'although', 'if', and 'unless'. Journal of Child Language, 8, 347-365.

RECEIVED: February 3, 1987; REvised: June 23, 1987. 